\title{
Nonlinear Modeling and Spectral Analysis of Ćuk Converters
}

\author{
K.T. Chau, C.C. Chan, and Jianming Yao \\ Department of Electrical and Electronic Engineering \\ The University of Hong Kong \\ Pokfulam, HONG KONG
}

\begin{abstract}
Being a fourth-order circuit, the Cuk converter certainly needs alone systematic studies of its nonlinear dynamics. In this paper, the investigation of the nonlinear dynamics of Cuk converters is carried out by deriving their nonlinear models and performing their spectral analyses. Nonlinear dynamics, including the additional dc offset, significant subharmonic and higher harmonic spectral contamination as well as the effects of self-intermodulation and crossintermodulation, due to large-signal perturbations of both the duty ratio and source voltage, are investigated.
\end{abstract}

\section{INTRODUCTION}

Due to the switching and nonlinear nature of power electronics circuits, the study of their dynamic behavior in both time and frequency domains has been one of the major research areas in power electronics [1]. The available timedomain approaches mainly focus on simulating the interested waveforms by using device-oriented simulation such as PSpice or circuit-oriented simulation. This time-domain simulation provides the transient behavior of power electronics circuits such as the maximum overshoot, rise time and settling time. On the other hand, the frequency-domain approaches have been widely based on deriving an approximated small-signal model of power electronics circuits. Incorporating superposition theorem with this smallsignal model, a set of transfer functions can be formulated to assess the dynamic behavior of those circuits even in the presence of multiple excitations. However, the small-signal model is valid only in the neighborhood of an operating point that is determined by the values of circuit parameters, supply voltage and load. Thus, the small-signal modeling techniques can neither handle large-signal perturbations nor depict system spectral contamination with subharmonic and harmonic frequency components.

The Volterra functional series (VFS) was first studied by Volterra in 1880's as a generalization of the Taylor series expansion of a function [2]. Starting from the early 1940's, the VFS has been successfully applied to the analysis of various nonlinear systems such as antennas, communications, fluid mechanics, biophysics and physiology [3]. In 1991, Tymerski firstly employed the VFS to investigate the controlto-output nonlinear frequency response of a pulse-width modulated (PWM) converter [4]. In 1993, Chau newly extended the VFS to investigate the nonlinear dynamics of various PWM converters in the presence of multiple excitations [5]. Recently, the VFS has also been successfully applied to PWM converters operating in the discontinuous conduction mode [6].

Since its invention in 1977 [7]-[9], the Cuk converter has received much attention from the arena of power electronics. Focus of attention has been around its design and use in the field of switch-mode power stages. Analysis of the Cuk converter has been successfully performed for the studies of steady-state behavior and small-signal dynamics. Although nonlinear modeling and spectral analysis of switch-mode power stages have recently been performed, the investigations have been based on those second-order converters - buck, boost and buck-boost [4]-[6]. Being a fourth-order circuit, the Cuk converter certainly needs alone systematic studies of its nonlinear dynamics.

It is the purpose of this paper to systematically investigate the nonlinear dynamics of Cuk converters by deriving their nonlinear models and performing their spectral analyses. Nonlinear dynamics, including the additional dc offset, significant subharmonic and higher harmonic spectral contamination as well as the effects of self- and crossintermodulation, due to large-signal perturbations of both the duty ratio and source voltage, will be investigated. The theoretical results will be verified by numerically comparing with the results obtained by PSpice simulation.

\section{VOLTERRA FUNCTIONAL SERIES}

The application of the VFS to problems in electrical engineering has been concentrated on single-input nonlinear systems such as antennas and communications. However, power electronics circuits are essentially nonlinear systems with dual inputs, such as the supply voltage and duty ratio for $\mathrm{dc}-\mathrm{dc}$ converters, as well as the supply voltage and delay angle for ac-dc converters. Thus, the conventional VFS needs to be extended in such a way that it can be applied to those power electronics circuits. In the followings, the VFS for single-input nonlinear systems is introduced first and then extended to dual-input nonlinear systems. The extension to other multiple-input nonlinear systems can similarly be performed with ever increasing complexity.

For single-input nonlinear systems, the output $y(t)$ can be expressed as a VFS of the input $u(t)$ : 
$y(t)=\sum_{n=0}^{\infty} y_{n}(t)$

$y_{n}(t)= \begin{cases}h_{0} & n=0 \\ \int_{-\infty}^{\infty} \cdots \int_{-\infty}^{\infty} h_{n}\left(\tau_{1}, \cdots, \tau_{n}\right) \prod_{i=1}^{n} u\left(t-\tau_{i}\right) d \tau_{i} & n>0\end{cases}$

where $y_{n}(t)$ and $h_{n}\left(\tau_{1}, \cdots, \tau_{n}\right)$ are called the $n$ th-order output and Volterra kernel, respectively. The multiple Laplace transform of this Volterra kernel becomes:

$H_{n}\left(s_{1}, \cdots, s_{n}\right)$

$= \begin{cases}h_{0} & n=0 \\ \int_{-\infty}^{\infty} \cdots \int_{\infty}^{\infty} h_{n}\left(\tau_{1} \cdots, \tau_{n}\right) \exp \left(-s_{1} \tau_{1}-\cdots-s_{n} \tau_{n}\right) d \tau_{1} \cdots d \tau_{n} & n>0\end{cases}$

where $H_{n}\left(s_{1}, \cdots, s_{n}\right)$ is called the $n$ th-order Volterra transfer function. Since this transfer function may not be unique in the sense that transfer functions with different permutations of the arguments can produce the same $n$ th-order output, it is usually symmetrized as follows:

$\bar{H}_{n}\left(s_{1}, \cdots, s_{n}\right) \equiv \frac{1}{n !} \sum_{p(\cdot)} H_{n}\left(s_{1}, \cdots, s_{n}\right)$

where $\rho(\cdot)$ denotes all possible permutations of the arguments. Since all Volterra transfer functions can readily be symmetrized, in the following, they will be assumed to be symmetric and the overbar will be omitted. Hence, the Volterra model of single-input nonlinear systems is shown in Fig. 1 .

For nonlinear systems with dual inputs $u_{a}(t)$ and $u_{b}(t)$, the output $y(t)$ can similarly be expressed as (1), but with different $n$ th-order output. Since the first few terms of the VFS are usually sufficient to represent the output, only the first three orders are derived. The higher orders can similarly be derived with ever increasing tedium. Firstly, the 0th-, 1st-, 2nd- and 3rd-order outputs are expressed as:

$$
\begin{aligned}
& y_{0}(t)=h_{0} \\
& y_{1}(t)=y_{1}^{a}(t)+y_{1}^{b}(t) \\
& =\sum_{n \in\{a, b\}} \int_{-\infty}^{\infty} h_{1}^{u}(\tau) u(t-\tau) d \tau \\
& y_{2}(t)=y_{2}^{a a}(t)+y_{2}^{b b}(t)+y_{2}^{a b}(t) \\
& =\sum_{u v \in\{a a, a b, b b\}} \int_{\infty}^{\infty} \int_{-\infty}^{\infty} h_{2}^{u v}\left(\tau_{1}, \tau_{2}\right) u\left(t-\tau_{1}\right) v\left(t-\tau_{2}\right) d \tau_{1} d \tau_{2} \\
& y_{3}(t)=y_{3}^{a a a}(t)+y_{3}^{b b b}(t)+y_{3}^{a a b}(t)+y_{3}^{a b b}(t) \\
& =\sum_{\substack{u, w \in \in \\
\{a a, b b b, a a b, a b b\}}} \int_{-\infty}^{\infty} \int_{-\infty}^{\infty} \int_{-\infty}^{\infty} h_{3}^{u v w}\left(\tau_{1}, \tau_{2}, \tau_{3}\right) v\left(t-\tau_{2}\right) w\left(t-\tau_{3}\right) d \tau_{1} d \tau_{2} d \tau_{3}
\end{aligned}
$$

where $a$ and $b$ indicate the contribution by $u_{a}(t)$ and $u_{b}(t)$, respectively. Notice that $y_{1}^{a}(t), y_{1}^{b}(t), y_{2}^{a a}(t), y_{2}^{b b}(t)$, $y_{3}^{a a a}(t)$ and $y_{3}^{b b b}(t)$ due to the individual contribution by either $u_{a}(t)$ or $u_{b}(t)$ are called the self-intermodulation outputs. On the other hand, $y_{2}^{a b}(t), y_{3}^{a a b}(t)$ and $y_{3}^{a b b}(t)$ due to the interaction by both $u_{a}(t)$ and $u_{b}(t)$ are called the cross-intermodulation outputs. By taking the multiple Laplace transform of those Volterra kernels, the corresponding Volterra transfer functions are given by:

$H_{0}=h_{0}$

$H_{1}^{u}(s)=\int_{\infty}^{\infty} h_{1}^{u}(\tau) \exp (-s \tau) d \tau$

$H_{2}^{u v}\left(s_{1}, s_{2}\right)=\int_{-\infty}^{\infty} \int_{-\infty}^{\infty} h_{2}^{u v}\left(\tau_{1}, \tau_{2}\right) \exp \left(-s_{1} \tau_{1}-s_{2} \tau_{2}\right) d \tau_{1} d \tau_{2}$

$H_{3}^{u v w}\left(s_{1}, s_{2}, s_{3}\right)=\int_{-\infty}^{\infty} \int_{-\infty}^{\infty} \int_{-\infty}^{\infty} h_{3}^{n v w}\left(\tau_{1}, \tau_{2}, \tau_{3}\right)$

where $u \in\{a, b\}, \quad u v \in\{a a, b b, a b\} \quad$ and $u v w \in$ $\{a a a, b b b, a a b, a b b\}$. Hence, the Volterra model of dualinput nonlinear systems can be constructed as Fig. 2.

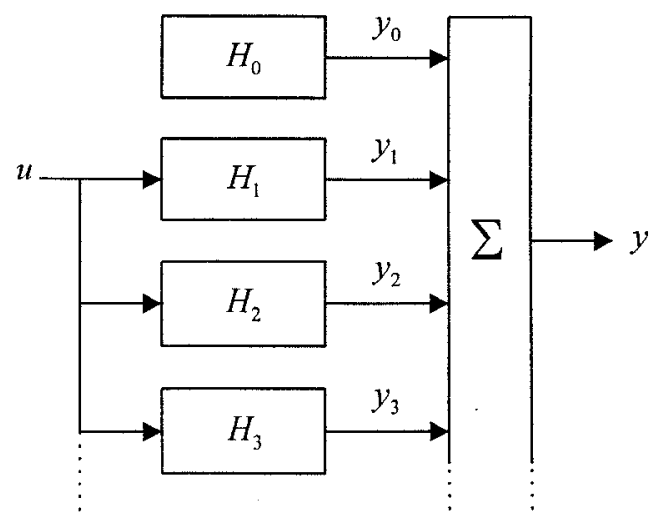

Fig. 1. Single-input Volterra model.

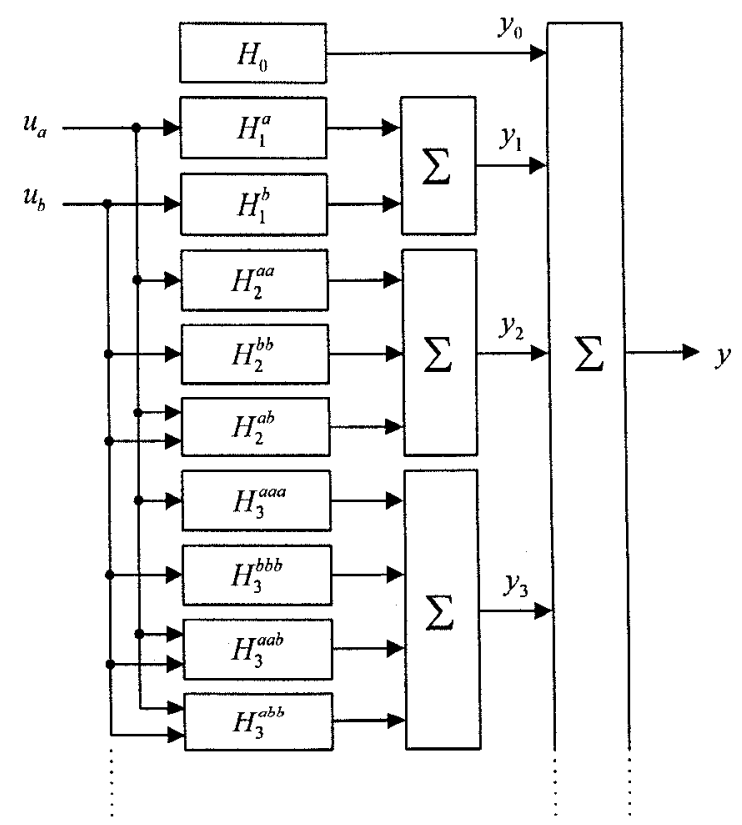

Fig. 2. Dual-input Volterra model. 
Since both inputs $u_{a}(t)$ and $u_{b}(t)$ may be composed of a number of frequency components, called the tones, whose amplitudes usually decrease with increasing frequencies, it is usually sufficient to consider the most significant two tones for the derivation. Thus, the general expression of these inputs are expressed as:

$$
\begin{aligned}
& u_{a}(t)=\left|A_{1}\right| \cos \left(\omega_{a 1} t+\angle A_{1}\right)+\left|A_{2}\right| \cos \left(\omega_{a 2} t+\angle A_{2}\right) \\
& u_{b}(t)=\left|B_{1}\right| \cos \left(\omega_{b 1} t+\angle B_{1}\right)+\left|B_{2}\right| \cos \left(\omega_{b 2} t+\angle B_{2}\right)
\end{aligned}
$$

By substituting (13), (14) into (6) and then using (10), the 1st-order output can be determined. Similarly, the 2nd-order output can be obtained by using (13), (14), (7) and (11), while the 3rd-order output is by using (13), (14), (8) and (12). The resulting 1st-, 2nd- and 3rd-order outputs are tabulated in Table I, where $A_{i}$ and $B_{i}$ are complex amplitudes of the ith tone of $u_{a}(t)$ and $u_{b}(t)$, respectively, $A_{i}^{*}$ and $B_{i}^{*}$ are their conjugates, and $A_{i}^{(*)}$ and $B_{i}^{(*)}$ are their optional conjugates corresponding to the minus sign of $\pm s_{a i}$ and $\pm s_{b i}$, respectively. Notice that when a negative frequency is encountered, the complex conjugation of its amplitude becomes a realistic one since $H_{n}\left(-s_{1}, \cdots,-s_{n}\right)=H_{n}^{*}\left(s_{1}, \cdots, s_{n}\right)$.

\section{NONLINEAR MODELING}

As shown in Fig. 3, the Cuk converter is of fourth-order. Since its natural frequency is generally well below the switching frequency, its operating behavior in the continuous conduction mode (CCM) can be formulated as:

$\dot{\mathbf{x}}=\left[\delta \mathbf{C}_{\mathbf{1}}+(1-\delta) \mathbf{C}_{2}\right] \mathbf{x}+\left[\delta \mathbf{D}_{1}+(1-\delta) \mathbf{D}_{2}\right] v_{s}$

where $\mathbf{x}=\left[\begin{array}{lllll}i_{L 1} & i_{L 2} & v_{C 1} & v_{C 2}\end{array}\right]^{\mathrm{T}}$ is called the state vector, $v_{s}$ is the supply voltage, $\delta$ is the duty ratio, and $\mathbf{C}_{1}, \mathbf{C}_{2}, \mathbf{D}_{1}, \mathbf{D}_{2}$ describing the topological stages are given by:

$\mathbf{C}_{1}=\left[\begin{array}{cccc}0 & 0 & 0 & 0 \\ 0 & 0 & 1 / L_{2} & -1 / L_{2} \\ 0 & -1 / C_{1} & 0 & 0 \\ 0 & 1 / C_{2} & 0 & -1 /\left(C_{2} R\right)\end{array}\right] \quad \mathbf{D}_{1}=\left[\begin{array}{c}1 / L_{1} \\ 0 \\ 0 \\ 0\end{array}\right](16)$
$\mathbf{C}_{2}=\left[\begin{array}{cccc}0 & 0 & -1 / L_{1} & 0 \\ 0 & 0 & 0 & -1 / L_{2} \\ 1 / C_{1} & 0 & 0 & 0 \\ 0 & 1 / C_{2} & 0 & -1 /\left(C_{2} R\right)\end{array}\right] \mathbf{D}_{2}=\left[\begin{array}{c}1 / L_{1} \\ 0 \\ 0 \\ 0\end{array}\right]$

By perturbing the variables in (15), and then separating the steady-state and perturbed quantities which are indicated with the overbar and tilde, respectively, both the steady-state and dynamic equations can be obtained as:

$$
\begin{aligned}
& \dot{\overline{\mathbf{x}}}=\left[\bar{\delta} \mathbf{C}_{1}+(1-\bar{\delta}) \mathbf{C}_{2}\right] \overline{\mathbf{x}}+\left[\bar{\delta} \mathbf{D}_{1}+(1-\bar{\delta}) \mathbf{D}_{2}\right] \bar{y}_{s}=0 \\
& \dot{\widetilde{\mathbf{x}}}=\mathbf{E}_{1} \widetilde{\mathbf{x}}+\mathbf{E}_{2} \widetilde{\delta}+\mathbf{E}_{3} \widetilde{v}_{s}+\mathbf{E}_{\mathbf{4}} \widetilde{\mathbf{x}} \widetilde{\delta}+\mathbf{E}_{5} \widetilde{\widetilde{v}_{s}} \widetilde{\delta}
\end{aligned}
$$

where $\mathbf{E}_{1}=\bar{\delta} \mathbf{C}_{1}+(1-\bar{\delta}) \mathbf{C}_{2}, \quad \mathbf{E}_{2}=\left(\mathbf{C}_{1}-\mathbf{C}_{2}\right) \overline{\mathbf{x}}+\left(\mathbf{D}_{1}-\mathbf{D}_{2}\right) \bar{v}_{s}$, $\mathbf{E}_{3}=\bar{\delta} \mathbf{D}_{1}+(1-\bar{\delta}) \mathbf{D}_{2}, \mathbf{E}_{\mathbf{4}}=\mathbf{C}_{1}-\mathbf{C}_{2}$, and $\mathbf{E}_{5}=\mathbf{D}_{1}-\mathbf{D}_{2}$.

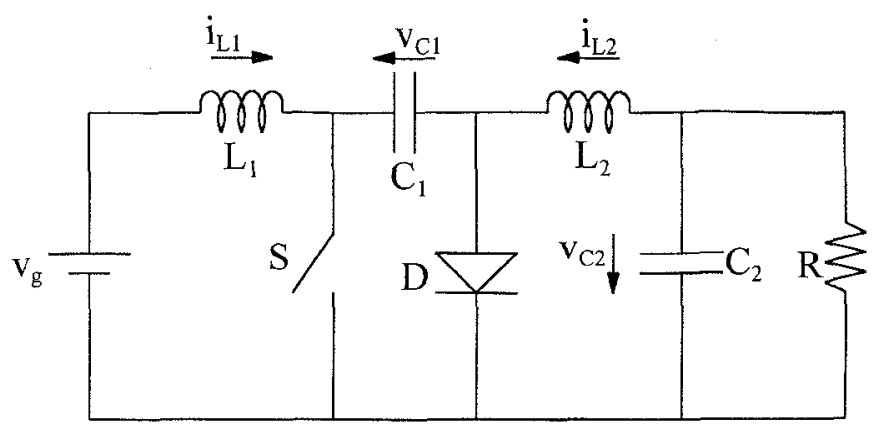

Fig. 3. Cuk converter.

TABLE I

THE 1ST-, 2ND- AND 3RD-ORDER OUTPUTS

\begin{tabular}{ll}
\hline Frequency & Amplitude \\
\hline$s_{a i}(i=1,2)$ & $A_{i} H_{1}^{a}\left(s_{a i}\right)$ \\
$s_{b i}(i=1,2)$ & $B_{i} H_{1}^{b}\left(s_{b i}\right)$ \\
$s_{a i} \pm s_{a i}(i=1,2)$ & $A_{i} A_{i}^{(*)} H_{2}^{a a}\left(s_{a j}, \pm s_{a i}\right)$ \\
$s_{b i} \pm s_{b i}(i=1,2)$ & $B_{i} B_{i}^{(*)} H_{2}^{b b}\left(s_{b i}, s_{b i}\right)$ \\
$s_{a i} \pm s_{a j}$ & $A_{i} A_{j}^{(*)} H_{2}^{a a}\left(s_{a i}, \pm s_{a j}\right)$ \\
$(i, j=1,2 ; i<j)$ & \\
$s_{b i} \pm s_{b j}$ & $B_{i} B_{j}^{(*)} H_{2}^{b b}\left(s_{b i}, \pm s_{b j}\right)$ \\
$(i, j=1,2 ; i<j)$ & \\
$s_{a i} \pm s_{b j}(i, j=1,2)$ & $0.5 A_{i} B_{j}^{(*)} H_{2}^{a b}\left(s_{a i}, \pm s_{b j}\right)$ \\
$3 s_{a i}(i=1,2)$ & $0.25 A_{i}^{3} H_{3}^{a a a}\left(s_{a i}, s_{a i}, s_{a i}\right)$ \\
$3 s_{b i}(i=1,2)$ & $0.25 B_{i}^{3} H_{3}^{b b b}\left(s_{b i}, s_{b i}, s_{b i}\right)$ \\
$s_{a i} \pm 2 s_{a j}$ & $0.75 A_{i} A_{j}^{* * 2)} H_{3}^{a a a}\left(s_{a i}, \pm s_{a j}, \pm s_{a j}\right)$ \\
$(i, j=1,2 ; i \neq j)$ & \\
$2 s_{a i}-s_{a i}(i=1,2)$ & $0.75 A_{i}^{2} A_{i}^{*} H_{3}^{a a a}\left(s_{a i}, s_{a i},-s_{a i}\right)$ \\
$s_{a i}-s_{a i}+s_{a j}$ & $1.5 A_{i} A_{i}^{*} A_{j} H_{3}^{a a a}\left(s_{a i},-s_{a i}, s_{a j}\right)$ \\
$(i, j=1,2 ; i \neq j)$ & \\
$s_{b i} \pm 2 s_{b j}$ & $0.75 B_{i} B_{j}^{(*) 2} H_{3}^{b b b}\left(s_{b i}, \pm s_{b j}, \pm s_{b j}\right)$ \\
$(i, j=1,2 ; i \neq j)$ & \\
$2 s_{b i}-s_{b i}(i=1,2)$ & $0.75 B_{i}^{2} B_{i}^{*} H_{3}^{b b b}\left(s_{b i}, s_{b i},-s_{b i}\right)$ \\
$s_{b i}+s_{b j}-s_{b j}$ & $1.5 B_{i} B_{j} B_{j}^{*} H_{3}^{b b b}\left(s_{b i}, s_{b j},-s_{b j}\right)$ \\
$(i, j=1,2 ; i \neq j)$ & $0.25 A_{i}^{2} B_{j}^{(*)} H_{3}^{a a b}\left(s_{a i}, s_{a i}, \pm s_{b j}\right)$ \\
$2 s_{a i} \pm s_{b j}(i=1,2)$ & $0.5 A_{i} A_{j}^{(*)} B_{k}^{(*)} H_{3}^{a a b}\left(s_{a i}, \pm s_{a j}, \pm s_{b k}\right)$ \\
$s_{a i} \pm s_{a j} \pm s_{b k}$ & \\
$(i, j, k=1,2 ; i<j)$ & $0.5 A_{i} A_{i}^{*} B_{j} H_{3}^{a a b}\left(s_{a i},-s_{a i}, s_{b j}\right)$ \\
$s_{a i}-s_{a i}+s_{b j}(i, j=1,2)$ & $0.25 A_{i} B_{j}^{(*) 2} H_{3}^{a b b}\left(s_{a i}, \pm s_{b j}, \pm s_{b j}\right)$ \\
$s_{a i} \pm 2 s_{b j}(i=1,2)$ & \\
$s_{a i} \pm s_{b j} \pm s_{b k} B_{j}^{* *} B_{k}^{(*)} H_{3}^{a b b}\left(s_{a i}, \pm s_{b j}, \pm_{b k}\right)$ \\
$(i, j, k=1,2 ; j<k)$ & $0.5 A_{i} B_{j} B_{j}^{*} H_{3}^{a b b}\left(s_{a i}, s_{b j},-s_{b j}\right)$ \\
$s_{a i}+s_{b j}-s_{b j}(i, j=1,2)$ & \\
\hline &
\end{tabular}


It should be noted that the solution of (17) represents the 0th-order Volterra transfer function which is identical to the relationship obtained by classical steady-state analysis. Increasingly, the last two terms in (18), which are the products of two perturbed variables, can mathematically represent the inherent nonlinearities of this converter.

In order to determine the 1st-, 2nd- and 3rd-order Volterra transfer functions, the inputs, namely the supply voltage and duty ratio, are generally represented by threeexponential expressions:

$$
\begin{gathered}
\widetilde{v}_{s}=u_{a}(t)=\sum_{i=1}^{3} \exp \left(s_{a i} t\right) \\
\widetilde{\delta}=u_{b}(t)=\sum_{i=1}^{3} \exp \left(s_{b i} t\right)
\end{gathered}
$$

while the output is expressed as:

$\widetilde{v}_{o}=y(t)=\mathbf{K} \widetilde{\mathbf{x}}$

where $K=\left[\begin{array}{llll}0 & 0 & 0 & 1\end{array}\right]$. By substituting (19)-(21) into (6)(8) and using (10)-(12), it can be found that the coefficients of those exponentials are the corresponding Volterra transfer functions. Thus, by substituting (19)-(21) into (18) and then equating those coefficients, the 1st-, 2nd- and 3rd-order transfer functions can be obtained as:

$$
\begin{aligned}
& H_{1}^{a}(s)=\mathbf{K}\left(s \mathbf{I}-\mathbf{E}_{\mathbf{1}}\right)^{-1} \mathbf{E}_{\mathbf{3}} \\
& H_{1}^{b}(s)=\mathbf{K}\left(s \mathbf{I}-\mathbf{E}_{\mathbf{1}}\right)^{-1} \mathbf{E}_{\mathbf{2}} \\
& H_{2}^{a a}\left(s_{1}, s_{2}\right)=0 \\
& H_{2}^{b b}\left(s_{1}, s_{2}\right)=\mathbf{K}\left[\left(s_{1}+s_{2}\right) \mathbf{I}-\mathbf{E}_{1}\right]^{-1} \mathbf{E}_{\mathbf{4}}\left(s_{1} \mathbf{I}-\mathbf{E}_{\mathbf{1}}\right)^{-1} \mathbf{E}_{2} \\
& H_{2}^{a b}\left(s_{1}, s_{2}\right)=\mathbf{K}\left[\left(s_{1}+s_{2}\right) \mathbf{I}-\mathbf{E}_{\mathbf{1}}\right]^{-1}\left[\mathbf{E}_{\mathbf{4}}\left(s_{2} \mathbf{I}-\mathbf{E}_{\mathbf{1}}\right)^{-1} \mathbf{E}_{\mathbf{3}}+\mathbf{E}_{5}\right] \\
& H_{3}^{a a a}\left(s_{1}, s_{2}, s_{3}\right)=0 \\
& H_{3}^{b b b}\left(s_{1}, s_{2}, s_{3}\right)= \mathbf{K}\left[\left(s_{1}+s_{2}+s_{3}\right) \mathbf{I}-\mathbf{E}_{1}\right]^{-1} \\
&\left.\mathbf{E}_{4}\left(s_{1}+s_{2}\right) \mathbf{I}-\mathbf{E}_{\mathbf{1}}\right]^{-1} \mathbf{E}_{\mathbf{4}}\left(s_{1} \mathbf{I}-\mathbf{E}_{1}\right)^{-1} \mathbf{E}_{\mathbf{2}} \\
& H_{3}^{a a b}\left(s_{1}, s_{2}, s_{3}\right)= 0 \\
& H_{3}^{a b b}\left(s_{1}, s_{2}, s_{3}\right)= \mathbf{K}\left[\left(s_{1}+s_{2}+s_{3}\right) \mathbf{I}-\mathbf{E}_{1}\right]^{-1} \\
& \mathbf{E}_{4}\left[\left(s_{1}+s_{3}\right) \mathbf{I}-\mathbf{E}_{\mathbf{1}}\right]^{-1}\left[\mathbf{E}_{\mathbf{4}}\left(s_{3} \mathbf{I}-\mathbf{E}_{\mathbf{1}}\right)^{-1} \mathbf{E}_{\mathbf{3}}+\mathbf{E}_{5}\right]
\end{aligned}
$$

Following the same procedure, the $n$ th-order transfer functions can be derived using $n$-exponential inputs. As indicated in (18), there is no interaction between the tones of $\widetilde{v}_{s}$ so that $H_{2}^{a a}=H_{3}^{a a a}=H_{3}^{a a b}=0$. Moreover, since the above transfer functions may not be symmetric, they are generally required to be symmetrized using (4).

\section{SPECTRAL ANALYSIS}

Based on the derived Volterra model of the Cuk converter, the investigation onto its nonlinear behavior is carried out. As shown in Fig. 3, the circuit parameters are selected as $L_{1}=L_{2}=200 \mu \mathrm{H}, \quad C_{1}=C_{2}=20 \mu \mathrm{F} \quad$ and $R=2 \Omega$ to ensure the converter operating in the CCM.

\section{A. Subharmonics, Harmonics and Dc Offset}

To investigate the nonlinear behavior of this converter, the supply voltage and duty ratio are selected as:

$$
\begin{aligned}
& v_{s}=20+4 \cos [2 \pi(600 t)]+2 \cos \left[2 \pi(700 t)+90^{\circ}\right] \mathrm{V} \\
& \delta=0.5+0.1 \cos \left[2 \pi(200 t)+180^{\circ}\right]+0.05 \cos \left[2 \pi(300 t)+270^{\circ}\right]
\end{aligned}
$$

Thus, $\bar{v}_{s}=20 \mathrm{~V}, \bar{\delta}=0.5,\left|A_{1}\right|=4 \mathrm{~V}, \angle A_{1}=0^{\circ},\left|A_{2}\right|=2 \mathrm{~V}$, $\angle A_{2}=90^{\circ}, \quad \omega_{a 1}=2 \pi(600) \mathrm{rad} / \mathrm{s}, \quad \omega_{a 2}=2 \pi(700) \mathrm{rad} / \mathrm{s}$, $\left|B_{1}\right|=0.1, \quad \angle B_{1}=180^{\circ}, \quad\left|B_{2}\right|=0.05, \quad \angle B_{2}=270^{\circ}$, $\omega_{b 1}=2 \pi(200) \mathrm{rad} / \mathrm{s}, \omega_{b 2}=2 \pi(300) \mathrm{rad} / \mathrm{s}$. By using (17), the 0th-order transfer function, and hence the steady-state $\mathrm{dc}$ output can be calculated. Then, by employing (22)-(30) and Table I, the 1st-, 2nd- and 3rd-order transfer functions, and hence the corresponding outputs can be calculated.

To illustrate the contribution and significance of each transfer function, the output voltage spectra resulting from using the combined 0th- and 1st-order transfer functions (Case A), the combined 0th-, 1st- and 2nd-order transfer functions (Case B), and the combined 0th-, 1st-, 2nd- and 3rd-order transfer functions (Case $\mathrm{C}$ ) are shown together in Fig. 4.

It can be seen that the spectrum for Case A, marked with crosses ( $x$ ), consists of the fundamental frequency components only, namely $200 \mathrm{~Hz}, 300 \mathrm{~Hz}, 600 \mathrm{~Hz}$ and $700 \mathrm{~Hz}$. It is due to the fact that the 1st-order output is identical to that obtained by using the small-signal modeling and superposition theorem. Thus, it can only roughly estimate the fundamental frequency components and is ill-suited for all other spectral components, namely those significant subharmonic components, $0 \mathrm{~Hz}$ and $100 \mathrm{~Hz}$, as well as harmonic components, such as $400 \mathrm{~Hz}, 500 \mathrm{~Hz}, 600 \mathrm{~Hz}, 800 \mathrm{~Hz}$ and $900 \mathrm{~Hz}$, which are caused by self- and crossintermodulation.

On the other hand, the spectrum for Case B, marked with pluses ( + ), is quite similar to that for Case $\mathrm{C}$, marked with circles $(O)$, except with some discrepancies which are due to the 3rd-order self- and cross-intermodulation components such as $(200-200+200)=200 \mathrm{~Hz}$ and $(200-200+600)=600 \mathrm{~Hz}$. It indicates that the contribution of the 3 -rd order output is not very significant, and focuses on the intermodulation components.

It is interesting to note that the $\mathrm{dc}$ component in the spectrum for Case $\mathrm{C}$ is significantly larger than that obtained from the 0th-order transfer function which is identical to the classical steady-state relationship $\bar{v}_{o}=\bar{v}_{s} \bar{\delta} /(1-\bar{\delta})$. This additional dc offset is mainly due to the 2 nd-order selfintermodulation components such as $(200-200)=0 \mathrm{~Hz}$ and $(300-300)=0 \mathrm{~Hz}$. Notice that these self-intermodulation components can be positive or negative, depending on the amplitudes and phase angles of those perturbations. 


\section{B. Inaccuracy of Small-Signal Modeling}

In order to illustrate the inaccuracy of using small-signal modeling to assess the output voltage at the fundamental frequencies, the percentage amplitudes of the perturbations with respect to their steady-state values are varied so that the supply voltage and duty ratio are given by:

$$
\begin{aligned}
& v_{s}=20\left\{1+2 k \cos [2 \pi(600 t)]+k \cos \left[2 \pi(700 t)+90^{\circ}\right]\right\} \quad \mathrm{V} \\
& \delta=0.5\left\{1+2 k \cos \left[2 \pi(200 t)+180^{\circ}\right]+k \cos \left[2 \pi(300 t)+270^{\circ}\right]\right\}
\end{aligned}
$$

where $k=0 \cdots 10 \%$ is defined as the perturbation factor. The errors of both the $\mathrm{dc}$ and fundamental frequency components obtained by using Case $\mathrm{A}$ with respect to those using Case $\mathrm{C}$ are shown in Fig. 5. It can be found that these spectral errors increase significantly with the perturbation factor. It also provides an indication on how large the amplitudes of perturbations can be allowed to employ the small-signal modeling. Focusing on this converter, the use of small-signal modeling is unacceptable even when the perturbation factor is $1 \%$. Of course, the spectral errors for other frequency components are always $100 \%$.

\section{Verification of Modeling Accuracy}

To verify the proposed approach, the modeling results are compared with the simulated results obtained by PSpice simulation. The experimental verification is not carried out because the modeling accuracy can hardly be proved in the presence of inevitable experimental errors. The PSpice simulation involves a tedious transient analysis from start-up to steady state, and a Fourier analysis of the resulting transient waveform over a defined period.

By using the same circuit parameters and the inputs given by (31) as well as selecting a switching frequency of $50 \mathrm{kHz}$, the PSpice-simulated waveform of its output voltage $v_{C 2}$ is shown in Fig. 6 . The corresponding waveforms of the inductor currents $i_{L 1}, i_{L 2}$ and the capacitor voltage $v_{C 1}$ are shown in Figs. 7 and 8 , respectively. Since $i_{L 1}, i_{L 2}$ and $v_{C 1}$ are always positive, the converter has been operating solely in CCM, never in the discontinuous conduction mode (DCM) nor in the discontinuous voltage mode (DVM).

The corresponding output voltage spectrum can be obtained by applying Fourier analysis to the simulated waveform ranging from $5 \mathrm{~ms}$ to $15 \mathrm{~ms}$. As shown in Fig. 9, the resulting spectrum, marked with crosses $(x)$, is compared with the previous modeling result, marked with circles $(O)$. As expected, the agreement between two spectra is very good. It should be noted that the PSpice-simulated spectrum is obtained at the expense of an hour, while the Volterra modeling spectrum can be obtained in less than a second. Moreover, numerical simulation can never provide an analytical model as given by the proposed approach.

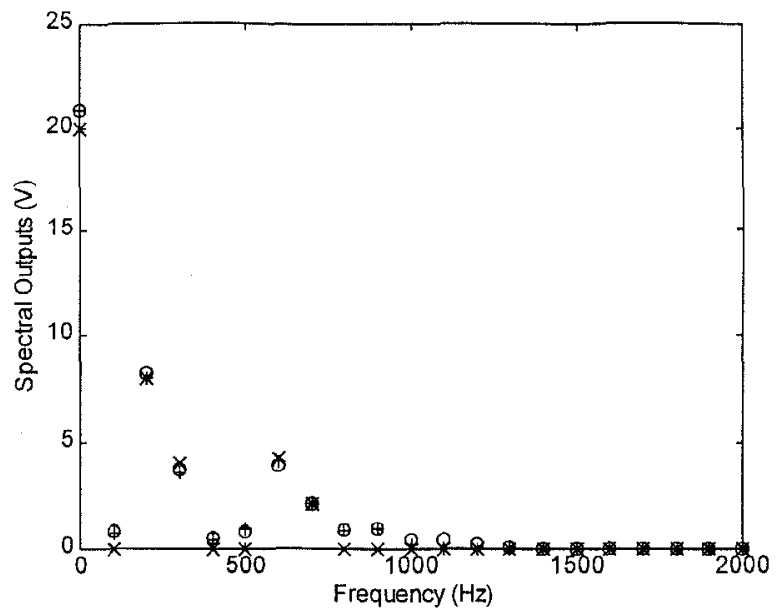

Fig. 4. Variations in spectral outputs. $\times$ : 0th- and 1st-order. +: 0th-, 1st- and 2nd-order. $O: 0$ th-, 1st-, 2nd- and 3rd-order.

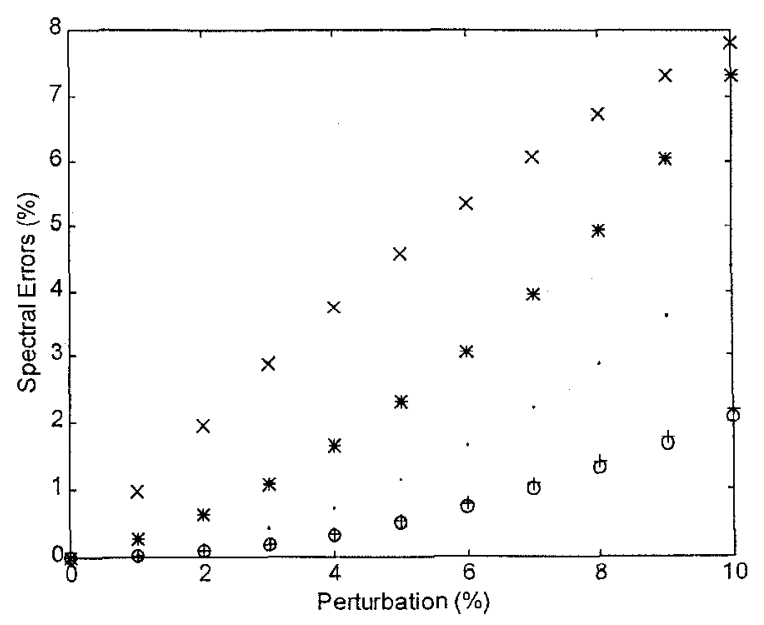

Fig. 5. Variations in spectral errors. $\because 0 \mathrm{~Hz} . \mathrm{O}: 200 \mathrm{~Hz} . \times: 300 \mathrm{~Hz}$. $: 600 \mathrm{~Hz} .+: 700 \mathrm{~Hz}$.

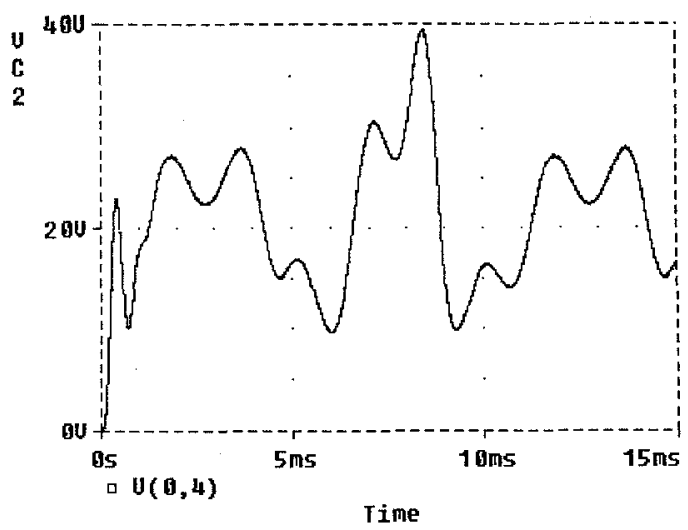

Fig. 6. Output voltage waveform resulting from PSpice simulation 


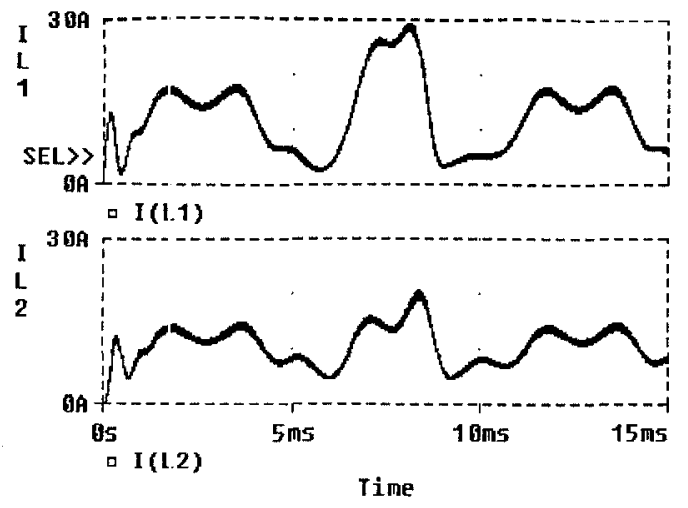

Fig. 7. Inductor current waveforms resulting from PSpice simulation.

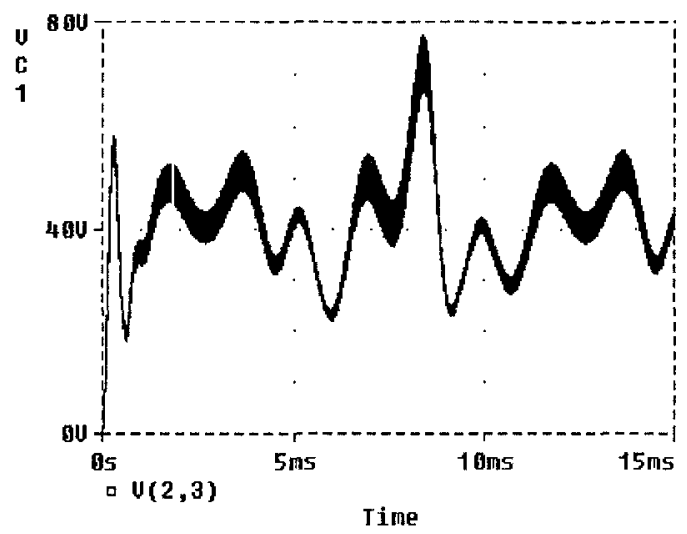

Fig. 8. Capacitor voltage waveform resulting from PSpice simulation.

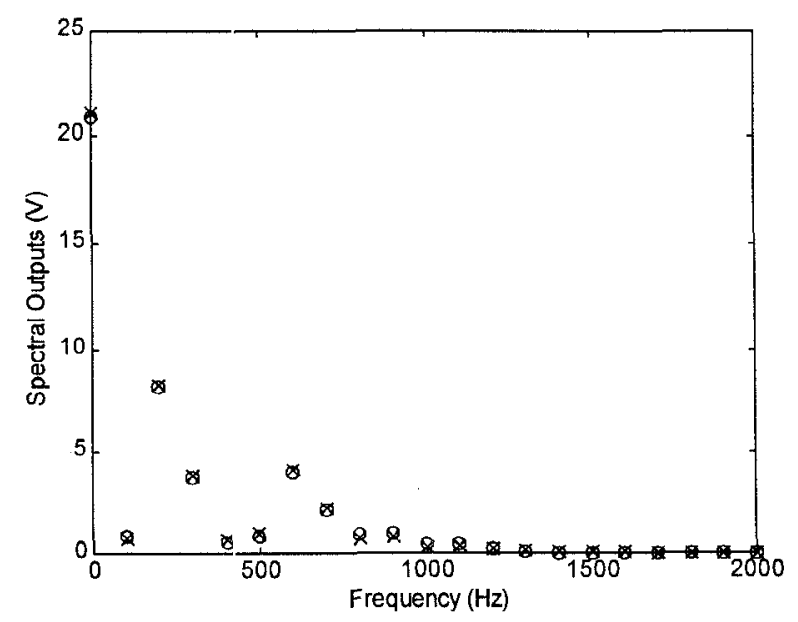

Fig. 9. Verification of spectral outputs. $x$ : PSpice simulation. $O$ : Volterra modeling.

\section{CONCLUSION}

By deriving the nonlinear models of Cuk converters and then performing their spectral analyses, the investigation onto their nonlinear dynamics has been carried out. The nonlinear dynamics include the additional $\mathrm{dc}$ offset, significant subharmonic and higher harmonic spectral contamination as well as the effects of self-intermodulation and crossintermodulation due to large-signal perturbations of both the duty ratio and source voltage. Although the investigation has only dealt with the Cuk converter operating in the CCM, the modeling and analysis for the operation in the DCM and DVM can similarly be carried out.

\section{ACKNOWLEDGMENT}

This work was supported and funded in part by the Committee on Research and Conference Grants, the University of Hong Kong.

\section{REFERENCES}

[1] F.C. Lee, Modeling, Analysis, and Design of PWM Converters. VPEC Publications Series, 1990.

[2] V. Volterra, Theory of Functionals and of Integral and Integro-Differential Equations. Dover, 1959.

[3] M. Schetzen, The Volterra and Wiener Theories of Nonlinear Systems. John Wiley \& Sons, 1980.

[4] R. Tymerski, "Volterra series modeling of power conversion systems," IEEE Trans. Power Electronics, vol. 6 , no. 4 , Oct. 1991 , pp. 712-718.

[5] K.T. Chau, "Nonlinear modeling of switching dc-dc converters with independ at inputs," Int. J. Electronics, vol. 75, no. 2, Apr. 1993, pp. 361-374.

[6] K.T. Chau, "Nonlinear modeling of switching dc-dc converters operating in discontinuous conduction mode," Int. J. Electronics, 1997.

[7] S. Cuk and R.D. Middlebrook, "A new optimal topology switching dc to dc converter," in Advances in SwitchedMode Power Conversion, vol. 2, TESLAco, 1983, pp. 311-330.

[8] S. Cuk, "Discontinuous inductor current mode in the optimal topology switching converter," in Advances in Switched-Mode Power Conversion, vol. 2, TESLAco, 1983, pp. 365-384.

[9] S. Cuk, "General topological properties of switching structures," in Advances in Switched-Mode Power Conversion, vol. 2, TESLAco, 1983, pp. 463-484. 